\title{
Severe anaphylactic reaction to patent blue in breast cancer surgery
}

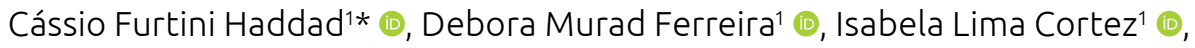

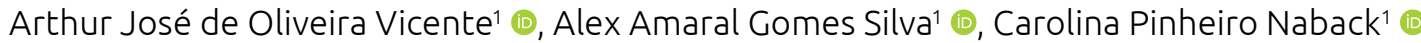

\section{ABSTRACT}

Sentinel lymph node biopsy in the surgical treatment of initial breast cancer has been increasingly adopted to assess axillary status as a way to replace total lymphadenectomy. The sentinel lymph node can be identified using coloring agents or radiopharmaceuticals. In Brazil, patent blue is the most used dye for this type of procedure, with high rates of identification and safety; however, in some cases, the use of this substance can lead to the occurrence of anaphylactic reactions. The case presented here refers to a 41-year-old female patient admitted for a surgical procedure for total mastectomy associated with sentinel lymph node biopsy with patent blue. After surgical initiation, the patient developed severe anaphylactic shock, staying 21 days in the intensive care unit (ICU) for recovery. Most anaphylactic reactions that occur in the transoperative period are mediated by IgE antibodies, resulting in the degranulation of mast cells and basophils, with the release of mediators such as histamine, prostaglandins, proteoglycans, and cytokines, leading to the clinical manifestations of anaphylaxis. There is evidence that part of the population is allergic to patent blue, and may present with manifestations ranging from hives and pruritus to severe cardiovascular collapse, requiring hospitalization in an ICU. The purpose of this article was to report a case of severe anaphylactic reaction to patent blue and to review the literature regarding this infrequent and potentially serious situation.

KEYWORDS: Anaphylaxis; Hypersensitivity; Sentinel lymph node biopsy; Breast neoplasms; Coloring agents.

\section{INTRODUCTION}

Sentinel lymph node biopsy (SLNB) in the surgical treatment of initial breast cancer has been widely used as part of the routine surgical protocol, avoiding total lymphadenectomy in most cases ${ }^{1}$. When breast cancer is metastasized, it usually spreads via the lymphatic pathway to the first lymph node affected by cancer cells, called the sentinel lymph node (SLN) ${ }^{2}$. The identification of this lymph node occurs through the use of coloring agents, such as patent blue, or radiopharmaceuticals, such as technetium, both used alone or in combination, or through indocyanine green. Patent blue is used by means of a subdermal injection in the breast, often in the periareolar region, gaining the lymphatic current, which, in turn, is drained almost entirely to the axillary region. The dye binds weakly to serum albumin and forms a complex that is captured by the afferent lymphatics, staining and identifying the SLN with a bright blue color ${ }^{1,3}$. The reaction to patent blue should also be considered in addition to the most frequently involved agents in perioperative hypersensitivity reactions - i.e. neuromuscular blockers, latex, and antibiotics - with an incidence between $0.07 \%$ to $2.7 \%^{3-6}$. Although rare, surgeons and anesthesiologists who perform the procedure with this type of mapping should be aware of possible adverse events and be prepared to manage them. This article aimed to report a case of severe anaphylactic reaction to patent blue used to identify SLN during breast cancer surgery, as well as to make a briefliterature review on this infrequent and potentially serious situation.

\section{CASE REPORT}

C.M.A., a female patient, aged 41 years old, admitted for mastectomy associated with SLNB and reconstruction with prosthesis. Carrier of chronic arterial hypertension, using propranolol and enalapril for control. Report of allergy to sulfa and sticking plaster. She was diagnosed with invasive carcinoma in the right breast, clinical stage cT4bN1M0, immunohistochemistry: positive ER, 
positive PR, negative Her-2, Ki67 37\% (Luminal B Her-2 negative), having been referred for neoadjuvant chemotherapy. After the end of the treatment, performed with 4 cycles of anthracycline and cyclophosphamide +4 cycles of taxane, uneventful and with good tolerance, she returned to the surgical schedule, presenting a complete clinical response. At mammography, a regression of the nodular lesion was observed, however, irregular microcalcifications were found, in a ductal path, in the central region of the right breast, extending from the anterior to the deep region. At breast ultrasound, the nodule previously described was not visualized and lymph nodes of usual appearance in axillary regions were identified. After case discussion, a skin-sparing mastectomy associated with SLNB and immediate reconstruction with prosthesis was indicated. Preoperative exams showed no changes, and the patient was classified as having surgical risk ASA II and was released for surgery under general anesthesia, after pre-anesthetic evaluation. During surgery, she received $2 \mathrm{~g}$ of cefazolin minutes before anesthetic induction, which was done with remifentanil in a continuous infusion pump (CIP), propofol (150 mg) and rocuronium (50 mg). She underwent orotracheal intubation with a $7.5 \mathrm{~mm}$ cuffed tube with no complications, and general anesthesia with remifentanil (CIP) and sevoflurane was maintained. The patient also received $10 \mathrm{mg}$ of IV dexamethasone during anesthetic induction. Finally, $4 \mathrm{ml}$ of patent blue were injected intradermally into the periareolar region of the right breast. After about 10 minutes, the surgical procedure was started and the patient developed severe edema in the ears, diffuse erythematous lesions in the upper limbs, chest (Figure 1)

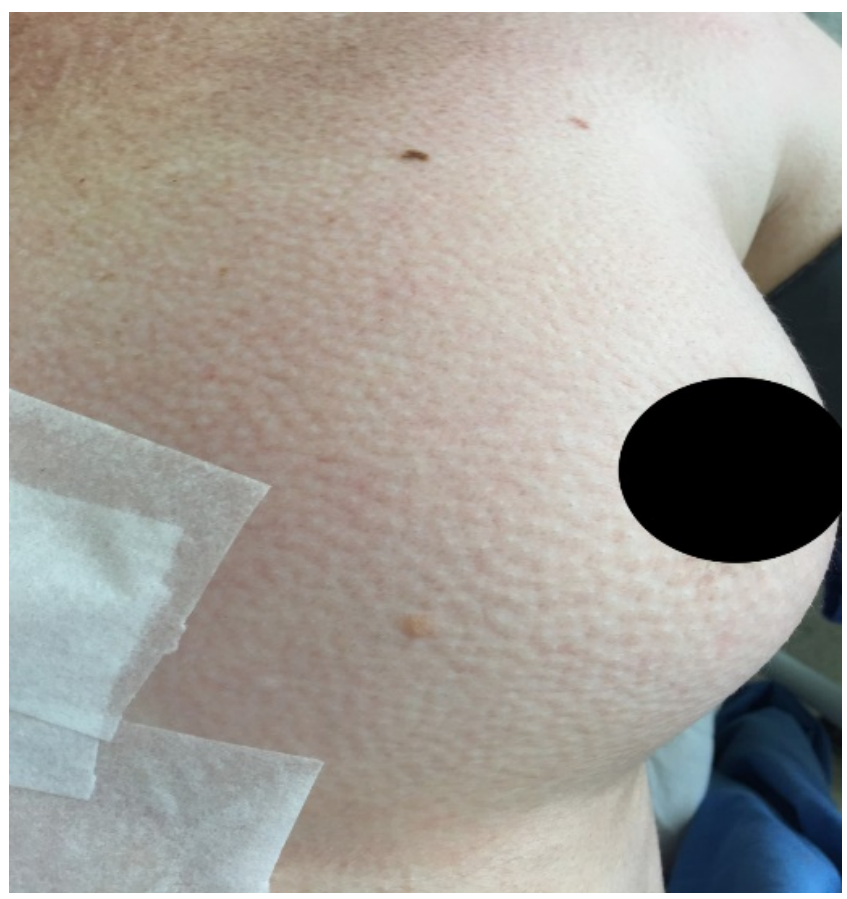

Figure 1. Erythema and goosebumps (cutis anserina) on the chest and left breast - right after the end of the surgical procedure. and abdomen, a sharp drop in the $\mathrm{CO}_{2}$ levels at the end of expiration $\left(\mathrm{ETCO}_{2}\right)$, volume-refractory hypotension, ephedrine, and phenylephrine. She presented an unsatisfactory response to adrenaline (500 mcg IV), with norepinephrine initiated in CIP, reaching $30 \mathrm{~mL} / \mathrm{h}$ to maintain satisfactory intra-arterial pressure (IAP), characterizing circulatory collapse. The woman did not present pulmonary auscultation compatible with bronchospasm. Anaphylactic shock was identified and hydrocortisone $500 \mathrm{mg} I V$ was administered. Then, total mastectomy with SLNB was performed, without the expected immediate reconstruction, due to the severity of the condition and the patient's hemodynamic instability. Postoperatively, the patient was referred to the intensive care unit (ICU), where she remained for 21 days, having spent 10 days intubated using vasoactive drugs and corticosteroids. After discharge from the ICU, she remained hospitalized for another 16 days, with motor deficit in upper and lower limbs and dysphonia, in addition to infection of the urinary tract by multi-resistant bacteria. A computed tomography scan of the skull was performed, which showed no changes, excluding stroke or local metastasis. After a total of 37 days of hospitalization, the patient was discharged for recovery at home, with physiotherapy and speech therapy, and for the sequence of adjuvant cancer treatment.

\section{DISCUSSION}

In Brazil, patent blue is the most used coloring agent for identifying SLN, followed, less frequently, by methylene blue, which showed an accuracy similar to that of patent blue in a randomized study ${ }^{7}$. The coloring agent can be administered by intradermal injection, as in the reported case, or intraparenchymatous injection, being captured by lymphatic vessels in the local drainage area and binding itself to albumin. Two thirds of it are absorbed in the first hour, being fully absorbed in 24 hours ${ }^{1,3}$. Excretion is done through urine and bile and the patient may observe blue-colored urine for 24 hours ${ }^{1,3}$. Usually, 0.5 to $4 \mathrm{ml}$ of dye are injected, the most used dose being $2 \mathrm{ml}$. Three degrees of severity related to hypersensitivity reactions to patent blue are described: grade I, corresponding to $69 \%-87 \%$ of cases, characterized by bluish hives, itching and generalized rash; grade II, corresponding to $3.2-8 \%$ of cases, presenting with hypotension (systolic blood pressure $<70 \mathrm{mmHg}$ ) without the need for vasopressors and absence of bronchospasm and laryngospasm; and grade III, corresponding to $1.1 \%$ of cases, characterized by severe cardiovascular collapse that requires a vasopressor, with suspension of the surgical procedure and transfer of the patient to the $\mathrm{ICU}^{1,3,8}$. In the present case, the patient had severe intraoperative anaphylactic reaction, which manifested as major edema in the ears, diffuse hyperemic lesions in the upper limbs, sudden drop in $\mathrm{ETCO}_{2}$, and refractory hypotension to medications, having to stay 21 days in the ICU for postoperative recovery. Thus, it 
was possible to classify the hypersensitivity reaction presented as grade III. Most anaphylactic reactions occurred during the operation are mediated by IgE antibodies and are potentially more severe than non-immunological anaphylactic reactions ${ }^{5}$. IgE-mediated anaphylaxis is caused by an IgE cross-reaction that results in mast cell and basophil degranulation. In a first exposure to the allergen, TH2 cells are activated, which stimulate the production of IgE antibodies. These, in turn, bind to receptors on the surface of mast cells and basophils, sensitizing these cells. Upon re-exposure to this allergen, binding to the $\operatorname{IgE}$ membrane receptor stimulates sensitized mast cells and basophils to degranulate. Degranulation intensely releases mediators such as histamine, prostaglandins, proteoglycans, and cytokines, leading to clinical manifestations of anaphylaxis ${ }^{4,9,10}$. Initial sensitization has no clinical manifestation. There is evidence that about $2.7 \%$ of the population would be allergic to the blue coloring agent, a situation attributed to the sensitization caused by repeated exposure to some products, such as fabrics, cosmetics, paper, leather, and medicines that contain these dyes ${ }^{1.5}$. Some risk factors for the development of anaphylaxis are also described, such as history of atopy, allergy to drugs or food, multiple surgeries, systemic mastocytosis, and hereditary angioedema ${ }^{5}$. It is noted that, in this case, the patient had a history of allergy to sulfa and adhesive tape. It is emphasized that skin changes are not always seen immediately, due to the presence of surgical drapes, and disappear within 1-20 hours. In addition, the onset of hypersensitivity occurs between 10-45 minutes after injection of the coloring agent (mean of 17 minutes) ${ }^{1,3}$. In this case, the symptoms started about 15 minutes after the administration of the patent blue. All drugs and antiseptics used in surgery should be investigated in an anaphylactic reaction study ${ }^{9,11}$. Provided the drugs used in the anesthetic act have low allergenic potential, the fact that the patient had already used the antibiotic elected for the prophylaxis of infection in surgery, the exclusion of latex reaction, and the onset of symptoms at about $15 \mathrm{~min}$ utes after the administration of patent blue, the diagnostic hypothesis was a severe anaphylactic reaction to patent blue. In severe cases, arterial blood gas analysis and renal and hepatic function tests are necessary to detect hypoperfusion lesions in these organs, in addition to tests that assist in the diagnosis of anaphylaxis, such as the measurement of plasma tryptase levels and the measurement of histamine ${ }^{3,10}$. Tryptase is found in mast cells in the forms $\alpha$-protriptase and $\beta$-tryptase. In normal situations, plasma $\alpha$-protriptase can be found, while $\beta$-tryptase is only released by mast cell granules during an allergic reaction. The level of total plasma tryptase measures both of them and can be elevated during anaphylaxis and in other conditions, such as myocardial infarction, amniotic fluid embolism, or trauma $a^{3,4}$. Histamine, on the other hand, reaches serum levels in 5 minutes, remaining elevated for only about 30 to 60 minutes in anaphylaxis, making it more advantageous to measure its urinary metabolites, such as methyl histamine, which is maintained up to 24 hours after the beginning of the episode ${ }^{10}$. In addition, the identification of possible allergens is an important aspect in the prevention of future anaphylaxis and can be performed through skin tests (prick and intradermal tests), measurement of specific serum IgE levels, or provocation tests ${ }^{4,5,11}$. Treatment in patients with anaphylactic reaction grades I and II is based on the use of corticosteroids (hydrocortisone, dexamethasone), antihistamines (diphenhydramine, promethazine), and volume replacement with crystalloid ${ }^{1}$. In patients classified as grade III anaphylactic reaction, there is a need for vasopressors (adrenaline, metaraminol, ethylene, ephedrine, and noradrenaline). The response to the vasopressor can be obtained with an initial dose or require prolonged infusion of the drug ${ }^{1,3}$. In this case, the patient presented refractory hypotension to volume and medication, in addition to an unsatisfactory response to adrenaline, with high-dose norepinephrine initiated, characterizing circulatory collapse. In view of the anaphylaxis, hydrocortisone was also administered. Total mastectomy with SLNB was performed, without the expected immediate reconstruction, due to the severity of the condition and the patient's hemodynamic instability, and it is recommended that the surgical procedure be completed as quickly and safely as possible in cases of perioperative anaphylaxis ${ }^{1,4}$. As reported, the patient remained in hospital for 37 days, 21 days in the ICU. In order to monitor patients, they must be admitted to the ICU in the postoperative period, since the condition can last up to 32 hours and biphasic reactions occur in up to $20 \%$ of cases. Fortunately, there are no cases of death described in the literature due to an allergic reaction to the use of patent blue ${ }^{1}$. Other ways of optimizing the prevention of this anaphylaxis were studied in addition to avoiding drugs whose tests were positive for hypersensitivity reactions, such as previous administration of glucocorticoids and antihistamines for procedures considered to be at high risk for anaphylaxis and the possibility that the use of lower coloring agent volumes can decrease allergic reactions. Further studies are needed to analyze the risk-benefit ratio of a prophylactic regimen, considering the low incidence of serious reactions and the possible adverse effects of steroids in particular ${ }^{12}$. An alternative to prevent an allergic reaction is to evaluate and test, preoperatively, each patient to confirm sensitivity to the blue dye. However, this approach, in addition to being questioned by the infrequency with which allergic reactions occur, is not so reliable, since false negative results are more likely to occur solely with a skin prick when compared to intradermal injection, which is more sensitive $^{4}$. In addition to prophylaxis, there are studies, although still inconclusive, on alternative methods of localizing SLN, such as the use of green indocyanine, which depends on the generation of molecular fluorescence, and the use of supermagnetic iron oxide (SPIO) nanoparticles, both without use of radiation and with lower risk of allergy, but dependent on technological 
acquisition and with difficult incorporation into the practice of most services in Brazil ${ }^{4}$. In a recent meta-analysis, Mok et al., comparing new SLN identification techniques, found superior results in terms of identification and false-negative rates with green indocyanine and SPIO nanoparticles compared to patent blue alone, and similar results when compared to the association of patent blue with technetium ${ }^{13}$. The already established use of radiopharmaceuticals and lymphoscintigraphy is not an accessible method to a considerable part of the surgical treatment centers for breast cancer, especially in the public health system, with the use of coloring agents being the option available for surgical identification of SLN.

\section{CONCLUSION}

Hypersensitivity reactions to patent blue are infrequent, but there is evidence that $2.7 \%$ of the population may be allergic to this type of dye. The manifestations presented can vary from hives to severe cardiovascular collapse. The reported patient had a grade III hypersensitivity reaction due to intradermal injection of patent blue. After treatment and 37 days of hospitalization, the patient progressed satisfactorily, being discharged for recovery at home. Therefore, it is extremely important that the entire medical team is aware of the possibility of an anaphylactic reaction occurring and able to identify and start treatment immediately.

\section{AUTHORS' CONTRIBUTIONS}

C.F.H.: Conceptualization, investigation, methodology, supervision, validation, visualization, writing - review \& editing. D.M.F.: investigation, validation, writing - review \& editing. I.L.C.: formal analysis, investigation, writing - original draft. C.P.N.: formal analysis, investigation, writing - original draft. A.A.G.S.: formal analysis, investigation, writing - original draft. A.J.O.V.: formal analysis, investigation, writing - original draft.

\section{REFERENCES}

1. Maranhão MVM, Nobrega DKA, Anunciação CEC, Maia BAB, Mariano PVD. Allergic reaction to patent blue dye in breast surgery [case report]. Rev Bras Anestesiol. 2016;66(4):433-6. http://doi.org/10.1016/j.bjane.2014.02.018

2. Wu HC, Yu JC, Yi LC, Wu CS, Lin SC, Wu ZF. Angioedema to patent blue dye in breast surgery: A case report and review of literatures. J Med Sci. 2019;39:43-8. http://doi.org/10.4103/ jmedsci.jmedsci_17_18

3. ShinzatoJY,MarcacciniACP,BragaAFA, RibeiroAR, RubioJEM, Souza JPD. Reação anafilática ao corante azul patente durante a biópsia do linfonodo sentinela em câncer de mama inicial - relato de caso. Rev Bras Ginecol Obstet. 2006;28(12):728-32. http://doi.org/10.1590/S0100-72032006001200007

4. Iqbal FM, Basit A, Salem F, Vidya R. Feeling blue, going green and finding other attractive alternatives: a case of biphasic anaphylaxis to patent blue and a literature review of alternative sentinel node localisation methods [case report]. BMJ. 2015;2015:bcr2015213107. http://doi.org/10.1136/bcr2015-213107

5. Faria E. Anaphylaxis to anesthetic agents [review article]. Rev Port Imunoalergologia. 2018;26(4):239-54.

6. Bézu C, Coutant C, Salengro A, Daraï E, Rouzier R, Uzan S. Anaphylactic response to blue dye during sentinel lymph node biopsy. Surg Oncol. 2011;20(1):e55-9. https://doi.org/10.1016/j. suronc.2010.10.002
7. Paulinelli RR, Freitas-Júnior R, Rahal RMS, Oliveira LFP, Vilela MHT, Moreira MAR, et al. A prospective randomized trial comparing patent blue and methylene blue for the detection of the sentinel lymph node in breast cancer patients. Rev Assoc Med Bras. 2017;63(2):118-23. http://doi.org/10.1590/1806-9282.63.02.118

8. Azevedo JP, Alves M, Ribeiro C, Bom AT. Anaphylactic shock perioperative to patent blue dye [case report].BMJ.2018;2018:bcr2018-224330. http://doi.org/10.1136/bcr-2018-224330

9. Brenet O, Lalourcey L, Queinnec M, Dupoiron D, Jayr C, Rosay H, et al. Hypersensitivity reactions to Patent Blue V in breast cancer surgery: a prospective multicentre study. Acta Anaesthesiol Scand. 2013;57(1):106-11. https://doi.org/10.1111/aas.12003

10. Menezes TT. Anafilaxia: diagnóstico e tratamento em unidade de pronto atendimento pediátrico. Bol Cient Pediatr. 2014;3(2):75-8.

11. Aurich S, Seitz AT, Janina T, Simon JC, Theudler R. A Case of Anaphylaxis to Patent Blue in a Patient with Sentinel Lymph Node Excision [case report]. Iran J Allergy Asthma Immunol. 2016;15(6):547-50.

12. Ortiz D, Alvikas J, Riker AI. A Case of Severe Anaphylactic Reaction Secondary to Isosulfan Blue Dye Injection. Ochsner J. 2015;15(2):183-6.

13. Mok CW, Tan SM, Zheng Q, Shi L. Network meta-analysis of novel and conventional sentinel lymph node biopsy techniques in breast cancer. BJS Open. 2019;3(4):445-52. https://doi. org/10.1002/bjs5.50157 